ORIGINAL ARTICLE

\title{
Long-term body weight outcomes of antidepressant-environment interactions
}

\author{
C Mastronardi ${ }^{1}$, GJ Paz-Filho' ${ }^{1}, \mathrm{E}$ Valdez ${ }^{2}$, J Maestre-Mesa ${ }^{2}$, J Licinio ${ }^{1}$ and M-L Wong ${ }^{1}$ \\ ${ }^{1}$ John Curtin School of Medical Research, Australian National University, Canberra, ACT, Australia and \\ ${ }^{2}$ University of Miami Miller School of Medicine, Miami, FL, USA
}

\begin{abstract}
Both obesity rates and antidepressant use have escalated in the last 20 years. Most people who start antidepressant treatment discontinue it on their own. Meanwhile, obesity rates continue to increase. To test the hypothesis that antidepressant use is a risk factor for obesity, even after long-term discontinuation, we developed a novel animal paradigm consisting of short-term exposure to stress and antidepressants, followed by long-term high-fat diet. We show here that recurrent restraint stress (RRS)-related weight loss is recovered 2 weeks after the end of stress in young growing rats receiving a high-fat diet. It is noteworthy that animals that received short-term antidepressant treatment with either imipramine or fluoxetine during 7 days of RRS showed behavioral evidence of antidepressant effects. When exposed to a highfat diet after stress and when antidepressant treatment had ended, the animals had significant increases in caloric intake, body weight (BW) and size from 17 to 22 weeks following antidepressant discontinuation when compared with (control) RRS animals treated with saline and fed with a high-fat diet. These data are consistent with the previously described phenomenon of time-dependent sensitization, and support the notion that enduring effects of short-term antidepressant treatment become manifest on a long-term basis after antidepressant discontinuation, during conditions of high stress followed by high-fat intake. Analyses of open field and body size measurements obtained in a small subset of animals show that animals previously exposed to antidepressant had no deficits in locomotor activity and were larger. Antidepressant exposure may therefore be a covert, insidious and enduring risk factor for obesity, even after discontinuation of antidepressant treatment. Our data support the concept of persistent, long-term effects of pharmacological-environment interactions on BW regulation.

Molecular Psychiatry (2011) 16, 265-272; doi:10.1038/mp.2010.122; published online 7 December 2010
\end{abstract}

Keywords: antidepressant; diet; restraint stress; body weight; body size; behavior

\section{Introduction}

Major depressive disorder (MDD) is a serious public health problem. Currently, the point prevalence of MDD is $\sim 4-7 \%$, and the lifetime prevalence estimate ranges from 15 to $20 \% .^{1,2}$ MDD is the leading cause of disability measured in years lost because of disability, and the largest single cause of nonfatal disease burden in Australia. ${ }^{3}$ It will become the second leading contributor to global burden of disease by the year 2020 (disability-adjusted life year). ${ }^{4}$ Approximately $59 \%$ of individuals with MDD seek help for their condition, and $35 \%$ receive medication or psychological treatment. ${ }^{3}$ According to the Canadian Community Health Survey, the prevalence of antidepressant

Correspondence: Professor J Licinio or Professor M-L Wong, John Curtin School of Medical Research, Australian National University, 131 Garran Road, Canberra, ACT 2601, Australia. E-mail: Julio.Licinio@anu.edu.au or mali.wong@anu.edu.au Received 1 September 2010; revised 14 October 2010; accepted 21 October 2010; published online 7 December 2010 use over a period of 12 months between 2001 and 2002 was estimated at $5.8 \% .^{5}$

MDD is a common complex disorder that affects 121 million people worldwide. In the United States, the economic burden of MDD is in the order of $\$ 100$ billions per year, with workplace costs being the largest component. ${ }^{6}$ Antidepressant dispensing has increased substantially during the last two decades in Western countries. In the United States, antidepressants are prescribed to 27 million people and they are the most frequently prescribed class of medication. ${ }^{7,8}$ In the United Kingdom, France and Australia, antidepressant prescriptions have increased substantially since the early 1990s with the entry of selective serotonin reuptake inhibitors (SSRIs) in the marketplace. ${ }^{9-11}$

Studies examining weight gain during longterm SSRI treatment have reported inconsistent results. The results of a large, cross-sectional study based on the General Electric Medical Records Database of MDD patients treated with antidepressant 
monotherapy for at least 1 year suggested differences in the proportion of patients who gained at least $7 \%$ of their body weight (BW) during treatment. The highest percentage of patients with weight gain was associated with mirtazapine (26\%), followed by the SSRIs (16-19\%). ${ }^{12}$ Antidepressant treatment can be effective in MDD, but compliance is low: in a large European study of 7525 patients, 56\% abandoned treatment within 4 months. ${ }^{13}$

It is generally accepted that the side effects such as weight gain can adversely affect adherence to therapy, but according to Bulloch and Patten, ${ }^{5}$ the main reason for non-adherence was forgetting $(74.5 \%$ of responders), followed by 'felt better' $(10.7 \%)$; side effects were reported as the fourth reason $(5.9 \%)$. It is commonly stated that patients return to their previous weight after they stop taking antidepressants, but this assumption is not evidence based.

To complicate matters, clinical and animal weight data during antidepressant treatment have been difficult to integrate because they appear to support divergent effects; a large body of studies have supported that administration of several antidepressants result in failure to gain weight or 'paradoxical' weight loss in rats, especially at high doses. ${ }^{14,15}$ Consequently, animal paradigms that help close some of these gaps could significantly expand our understanding of the interface between obesity and MDD. There is a strong body of translational work that uses rodents to study the biology of depression and antidepressants. These studies have shown that a mechanism of action of antidepressants is to promote neurogenesis in the adult rat hippocampus. ${ }^{16}$ Animal models of depression have included stress paradigms (such as uncontrollable stress, chronic mild stress and repeated restraint stress (RRS)) that have been shown to decrease cell proliferation in the hippocampus, and administration of antidepressants can block this downregulation of cell proliferation. ${ }^{17}$

The use of antidepressants has grown dramatically since the late 1980s with the advent of the selective monoamine reuptake inhibitors. ${ }^{27}$ Vast numbers of people are exposed to antidepressants on a shortterm basis, as long-term compliance is not usually achieved. ${ }^{3,5}$ Such increased exposure to even short courses of antidepressant drugs temporally coincides with the emerging epidemic of obesity that is faced by developed countries. Could the current dramatic increase in obesity be attributed at least in part to exposure to antidepressants? It has been previously demonstrated that the effects of drugs may continue to increase over time, even after a single dose and as drug levels decrease: this intriguing phenomenon is known as time-dependent sensitization (TDS). ${ }^{18}$

We have hypothesized that TDS, which has been described during short-term antidepressant treatment, may apply to weight regulation after exposure to antidepressants. To test this hypothesis, we developed an animal paradigm that combines RRS and behaviorally effective, short-term antidepressant treatment, followed by long-term high-fat diet. This mimics a clinical situation experienced by millions of people: stress/depression is associated with short-term exposure to antidepressants and with long-term ingestion of high-fat diets. In such a paradigm, we tested the specific hypothesis that even short-term exposure to antidepressants represents a long-term risk factor for obesity, manifested protractedly when unmasked by environmental factors, such as high-fat diet.

\section{Materials and methods}

\section{Animals}

All procedures were performed under established guidelines of humane care and use of rats, and were approved by the University of Miami Institutional Animal Care and Use Committee, and by the Australian National University Ethics Committee. Upon arrival, virus- and antibody-free young adult male Sprague-Dawley rats (Harlan, Indianapolis, IN, USA) were housed at $24^{\circ} \mathrm{C}$ and $12 \mathrm{~h}$ light/dark schedule (lights on from 0600 to $1800 \mathrm{~h}$ ) in a stressfree environment and divided into two studies: (1) chronic antidepressant treatment and (2) stress, antidepressant and diet (hereafter, stress-antidepressant-diet study).

\section{Chronic antidepressant treatment study}

Young growing rats (150-200 g) were housed two per cage in a stress-free environment for at least 5 days before the initiation of experimental procedures. Rats were randomly assigned to two experimental groups: control ( $0.9 \%$ saline; Hospira, Lake Forest, IL, USA), $n=10$ and fluoxetine $10 \mathrm{mg}$ (SSRI, Sigma-Aldrich, St Louis, MO, USA), $n=10$. Animals received daily $0.5 \mathrm{ml}$ intraperitoneal injections of either $0.9 \%$ saline or fluoxetine $10.0 \mathrm{mg} \mathrm{kg}^{-1}$ dissolved in $0.9 \%$ saline, for 5 weeks. Dose and treatment duration were based on previous reports. ${ }^{19-21}$ BW was measured weekly.

\section{Stress-antidepressant-diet study}

Rats (200-230g) were housed one per cage (11" wide $\times 8.5^{\prime \prime}$ height $\times 14.5^{\prime \prime}$ long). Food intake (FI) and BW were measured several times a week starting one day after arrival (experimental day 1). Rats were given ad libitum access to food and water, except during the RRS sessions. This experiment lasted 177 days.

Rats were randomly assigned to two main groups: (1) non-restrained control (non-RRS) group $(n=26)$ and (2) restrained (RRS) group $(n=38)$. Animals in the non-RRS group were not injected or restrained, and this group comprised two subgroups: (i) NR-CC $(n=13)$, comprising non-RRS animals fed with regular chow diet throughout the whole experiment; and (ii) NR-CF $(n=13)$, comprising non-RRS animals fed with chow diet until day 11, and fed with adjusted fat diet thereafter (TD95217; Harlan, Saint Louis, MO, USA).

Animals in the RRS group were subjected to RRS as described below, and received adjusted fat diet (TD95217; Harlan) after day 11. The RSS group 
comprised two subgroups: (i) R-C group $(n=13)$, comprising RRS animals that received once daily intraperitoneal injection of $0.5 \mathrm{ml}$ of saline $(0.9 \%$ $\mathrm{NaCl}$; Hospira); and (ii) R-AD group $(n=25)$, comprising RRS plus antidepressant-treated animals receiving daily intraperitoneal injection of antidepressants for 7 days during the RRS period. Those animals received imipramine (Sigma-Aldrich) $10 \mathrm{mg} \mathrm{kg}^{-1}$ (RIMI, $n=13$ ) or fluoxetine (Sigma-Aldrich) $10 \mathrm{mg} \mathrm{kg}^{-1}$ (R-FLX, $n=12$ ).

Repeated restraint stress. We used flat-bottom clear acrylic restrainers $(20.3 \times 8.3 \mathrm{~cm})$ (Cat no. 544-RR; Plas Labs, Lansing, MI, USA). RRS sessions occurred during the period of 0900 to $1600 \mathrm{~h}$ and lasted $6 \mathrm{~h}$ each; they occurred for 7 consecutive days (days 5-11).

$B W$ and FI. During the RRS period, BW and FI were measured daily in all animals; BW gain was calculated as the area under the BW curve for nonRRS $(n=26)$ or RSS $(n=38)$ groups between days 5 and 11 (Figure 2b). After the restraining period, BW and FI were recorded three times per week until the end of the study at day 177 .

Determination of absolute cumulative caloric intake: FI was assessed by calculating the weight difference of the food pellets remaining on the cage top between two consecutive determinations. The amount of food consumed was multiplied by its respective caloric content $\left(3.36 \mathrm{kcal} \mathrm{g}^{-1}\right.$ for the regular chow and $4.3 \mathrm{kcalg}^{-1}$ for the adjusted fat diet). The mean cumulative caloric intake was calculated separated for the restraint period for the non-RRS and the RRS groups. The cumulative intake during the post-restraint period (between days 12 and 177) was plotted as a function of time for the two major restrained groups, namely R-C and R-AD.

Daily caloric intake: Daily caloric intake was calculated between days 133 and 163, a period including a total of 14 periods. Individual FI increments were converted into daily caloric intake by dividing the total amount of calories determined between two consecutive measurements by the day interval ( 2 or 3 days). These individual caloric intake values were averaged for each period for each experimental group (R-C and R-AD).

Linear growth, fat mass and behavior. Body and bone length and locomotor activity were determined in a subset of rats (R-C, $n=5$; R-IMI, $n=5$; and R-FXT, $n=4)$.

Body measurements: Ano-nasal length was determined weekly (four times) between days 133 and 150 .

Bone measurements: At the end of the study (day 177), rats were euthanized and their left hind legs were also dissected and heated for $2 \mathrm{~h}$ at $80^{\circ} \mathrm{C}$ in a solution containing $3 \mathrm{ml}$ ammonia solution $(7 \mathrm{~N}$ in methanol) and $25 \mathrm{ml}$ of water to facilitate bone dissection. Lengths of dissected femurs and tibias were measured with a caliper.
Fat mass: At the end of the study (day 177), rats were euthanized and epididymal fat pads were dissected and their fresh weights were obtained.

Locomotor activity: During the post-restraint period (14-67 days), a subset of rats (R-C, $n=5$ and R-AD, $n=9$ ) was submitted to 7 weekly $60 \mathrm{~min}$ open field test sessions (between 1400 and $1600 \mathrm{~h}$ ). Animals were individually placed in clear acrylic boxes $(40.64 \times 40.64 \mathrm{~cm})$ equipped with Digiscan activity monitors (Omnitech Electronics, Columbus, $\mathrm{OH}$, USA) with infrared light-sensitive detectors situated $2.5 \mathrm{~cm}$ apart on two perpendicular walls. Located along the opposing wall were infrared light beams directed at the detectors. One count of horizontal activity was registered each time the animal intercepted the beam. The total distance (TD) was obtained as horizontal activity counts and the center distance (CD) was obtained as horizontal activity counts in the center of the box. In each session, TD, CD and the ratio $\mathrm{CD} / \mathrm{TD}$, used as an index of anxiety, were calculated for the R-C and the R-AD groups. Their means were subsequently averaged along the seven sessions.

\section{Statistical analysis}

Differences among $\geqslant 3$ groups were analyzed by oneway analysis of variance followed by the StudentNeuman-Keuls multiple comparison test for unequal replications. Differences between two groups were analyzed by ' $t$ ' test or Mann-Whitney test when appropriate. The significance level for each of these effects was set at $P<0.05$. All statistical analyses were performed using GraphPad Prism 5.00 (GraphPad Software, La Jolla, CA, USA).

\section{Results}

Effects of chronic antidepressant treatment on BW Stress-free animals receiving regular chow and treated with chronic administration of fluoxetine $10 \mathrm{mg} \mathrm{kg}^{-1}$ for 5 weeks had lower weight when compared with animals treated with saline (mean \pm s.e., $336.7 \pm 3.8 \mathrm{~g}$ for saline and $312.0 \pm 3.7 \mathrm{~g}$ for fluoxetine, $P<0.0001$ ).

\section{Effects of stress-antidepressant-diet paradigm}

Figure 1a shows BW changes for all the five groups during the entire duration of the stress-antidepressant-diet study.

Acute effects of RRS. RRS sessions were performed for 7 consecutive days (days 5-11); during the RRS sessions, rats that were submitted to RRS ingested less calories (mean \pm s.e., $672.6 \pm 9.5$ and $753.1 \pm 15.7 \mathrm{kcal}$, respectively, for RRS and non-RRS groups, $P<0.0001$, Figure 2a) and gained significantly less weight $(1486.0 \pm 6.4 \mathrm{~g} \times$ day for RSS and 1592.0 $\pm 13.5 \mathrm{~g} \times$ day for non-RSS, $P<0.0001$, Figure 2b) when compared with control non-stressed animals.

Fat diet. Adjusted fat diet was initiated on study day 11 and caused non-stressed animals (NR-CF) to 


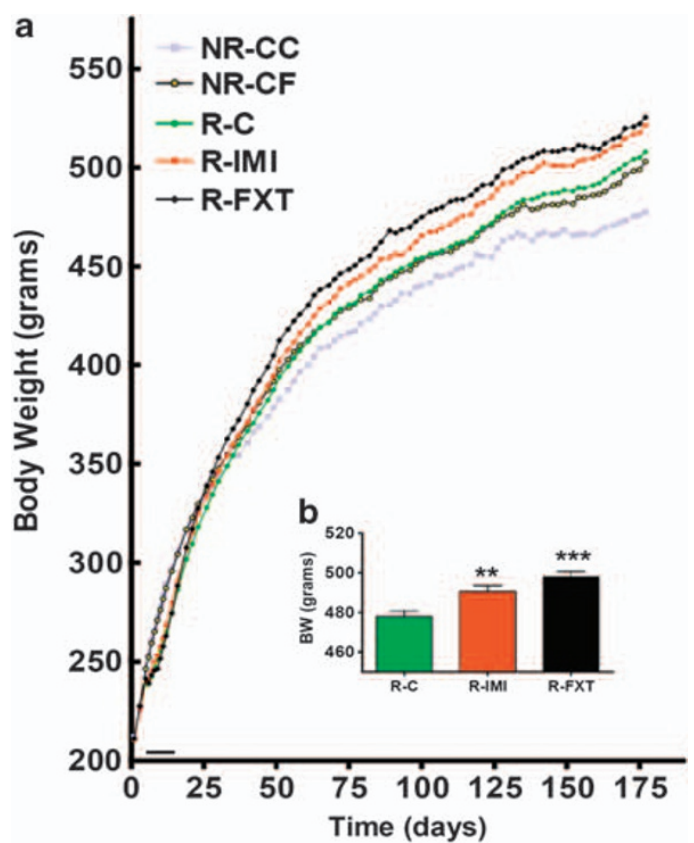

Figure 1 Body weight (BW) in grams during the experimental period. (a) BW was measured during days 1-177 of our stress-antidepressant-diet study. The small black bar above the $x$ axis indicates the recurrent restraint stress (RRS) period, $6 \mathrm{~h} \mathrm{day}^{-1}$ during 1 week. Dots represent mean BW at each particular day. NR-CC, non-restraint control on regular diet group $(n=13)$; NR-CF, non-restraint control on fat diet group ( $n=13)$; R-C, untreated RRS on fat diet group $(n=13)$; R-IMI, imipramine-treated RRS on fat diet group $(n=13)$; R-FXT, fluoxetine-treated RRS on fat diet group $(n=12)$. The inset (b) depicts the average BW of the three experimental groups subjected to RRS and fed with high-fat diet during the post-restraint period. Columns and bars depict mean and the s.e.m., respectively. Antidepressant treated groups were compared to control R-C group. ${ }^{*} P<0.01 ;{ }^{*}{ }^{*} P<0.001$.
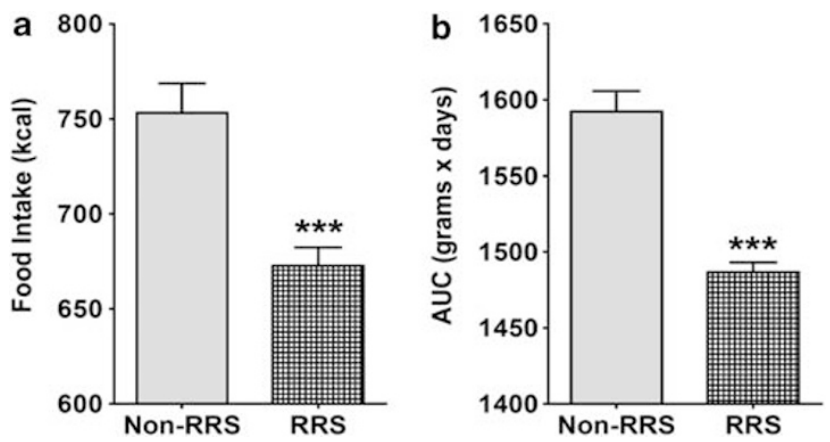

Figure 2 Interactions of recurrent restraint stress (RRS) and short-term antidepressant treatment on food intake and body weight during the RRS sessions. (a) Cumulative absolute intake (kcal) during the restraint stress (days 5-11) period in non-RRS $(n=26)$ and RRS rats $(n=38)$; during this period, all animals were fed with regular chow. Non-RRS consumed a larger amount of kcal. (b) Body weight gain was calculated by obtaining the area under the weight curve (AUC, $g \times$ day) during the restraining period. Non-RRS animals were heavier than RRS ones. ${ }^{* * *} P<0.0001$. become heavier than those receiving regular chow (NR-CC) (503.0 \pm 8.5 and $477.4 \pm 6.5 \mathrm{~g}$, respectively, $P=0.025)$.

Post-stress recovery period. In the immediate poststress period, stressed and non-stressed animals fed with high-fat diet had similar absolute caloric intake. RSS animals that received saline (R-C) and fed with high-fat diet achieved full weight recovery, and their weights at day 26 were not significantly different from those of non-stressed animals (NR-CF) receiving fat diet $(328.0 \pm 2.7$ and $336.8 \pm 4.8 \mathrm{~g}$, respectively, $P=0.1)$. Late in the post-stress recovery period (133-163 days), antidepressant-treated rats (R-AD) became heavier than R-C (Figure 1b, $498.0 \pm 2.71 \mathrm{~g}$ for R-FXT, $490.4 \pm 3.06 \mathrm{~g}$ for R-IMI and $477.8 \pm 2.85 \mathrm{~g}$ for R-C, $P<0.001$ for R-FXT and R-C; and $P<0.01$ for R-IMI and R-C) and had significantly higher caloric intake when compared with the R-C group (Figures 3a and b, 70.9 \pm 1.3 for R-AD and $66.0 \pm 1.0$ for R-C, $P=0.006$ ). During this period both $\mathrm{R}-\mathrm{AD}$ and R-C groups were fed with high-fat diet.

\section{Linear growth, fat mass and behavior.}

Linear growth: Compared with the non-treated RSS animals, antidepressant-treated RRS animals had larger body size (Figure 3c, $27.3 \pm 0.2 \mathrm{~cm}$ for R-AD $(n=9)$ and $26.0 \pm 0.4 \mathrm{~cm}$ for R-C $(n=5), P=0.009)$, longer femur (Figure 3d, $4.2 \pm 0.01 \mathrm{~cm}$ for R-AD and $4.1 \pm 0.03 \mathrm{~cm}$ for R-C, $P=0.01$ ) and tibia (Figure 3e, $4.5 \pm 0.02 \mathrm{~cm}$ for $\mathrm{R}-\mathrm{AD}$ and $4.4 \pm 0.03 \mathrm{~cm}$ for $\mathrm{R}-\mathrm{C}$, $P=0.004)$.

Epididymal fat pad measurements: As expected, fat diet increased total body fat content as measured by the fresh weight of epididymal fat pads $(12.6 \pm 0.7 \mathrm{~g}$ for NR-FC and $7.4 \pm 1.0 \mathrm{~g}$ for NR-CC, $P=0.001$ ) and epididymal fat pad/BW ratios $\left(23.3 \pm 0.9 \times 10^{-3}\right.$ for NR-FC and $14.9 \pm 1.8 \times 10^{-3}$ for NR-CC, $P=0.0003$ ), but these parameters were not significantly different between stressed and non-stressed animals (12.8 \pm $0.7 \mathrm{~g}$ for RRS and $11.9 \pm 1.7 \mathrm{~g}$ for non-RRS, $P=0.53$; $23.4 \pm 0.9 \times 10^{-3}$ for RRS and $22.8 \pm 2.4 \times 10^{-3}$ for non-RRS, $P=0.8$ ).

Locomotor Activity: A subset of antidepressanttreated animals was tested for locomotor activity and they showed higher locomotor activity reflected both as TD (Figure 4a, $4011.0 \pm 187.4 \mathrm{~cm}$ for R-AD and $2824.0 \pm 324.7 \mathrm{~cm}$ for R-C, $P=0.003$ ) and CD (Figure $4 \mathrm{~b}, 1375.0 \pm 58.8 \mathrm{~cm}$ for R-AD and $779.9 \pm 66.3 \mathrm{~cm}$ for R-C, $P<0.0001$ ), and the ratio $\mathrm{CD} / \mathrm{TD}$ suggests that RSS animals treated with antidepressants were less anxious in the open field test in comparison with non-treated RRS animals (Figure 4c, $0.4 \pm 0.01$ for R$\mathrm{AD}$ and $0.3 \pm 0.01$ for R-C, $P=0.004)$.

\section{Discussion}

We show here that during the course of a stress paradigm (restraint), short-term exposure to antidepressants decreases the depressive and anxiety behavioral correlates of stress. However, that short exposure to antidepressants is associated with 

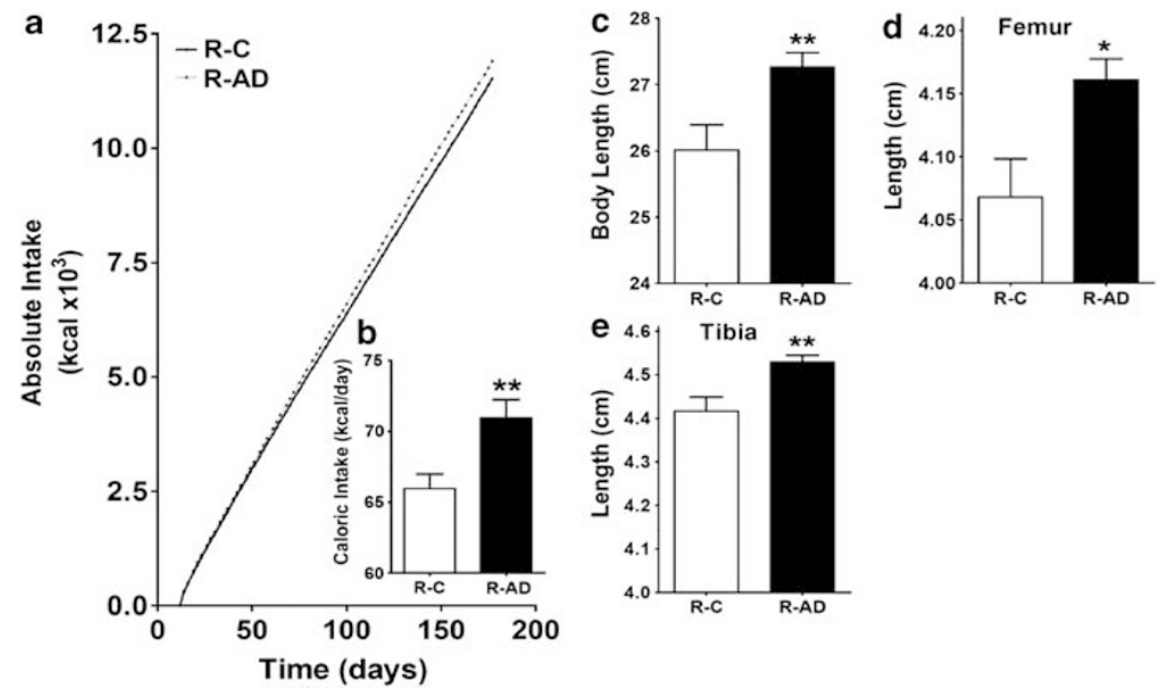

Figure 3 Interactions of recurrent restraint stress (RRS), short-term antidepressant treatment and fat diet on caloric intake, body weight and bone length. (a) Cumulative absolute intake $\left(\mathrm{kcal} \times 10^{3}\right)$ during post-restraint stress (days 12-177). Late in the post-stress recovery period (starting on day 133 or 4.4 months), antidepressant-treated animals had significantly higher caloric intake when compared with non-treated RRS animals (R-C, $n=13$; R-AD, $n=25)$. Inset (b) shows the average of daily caloric intake (days 133-163) for 14 periods. (c) Body length (cm) at day 150; R-C, $n=5$; R-AD, $n=9$. Antidepressant-treated RRS had larger bodies. (d) Femur length (cm) was obtained at end of the experiment, day 177. Antidepressant-treated RRS animals had longer femurs. (e) Tibia length was obtained at day 177. Antidepressant-treated RRS animals had longer tibias. ${ }^{*} P<0.05 ;{ }^{*} P<0.01$.
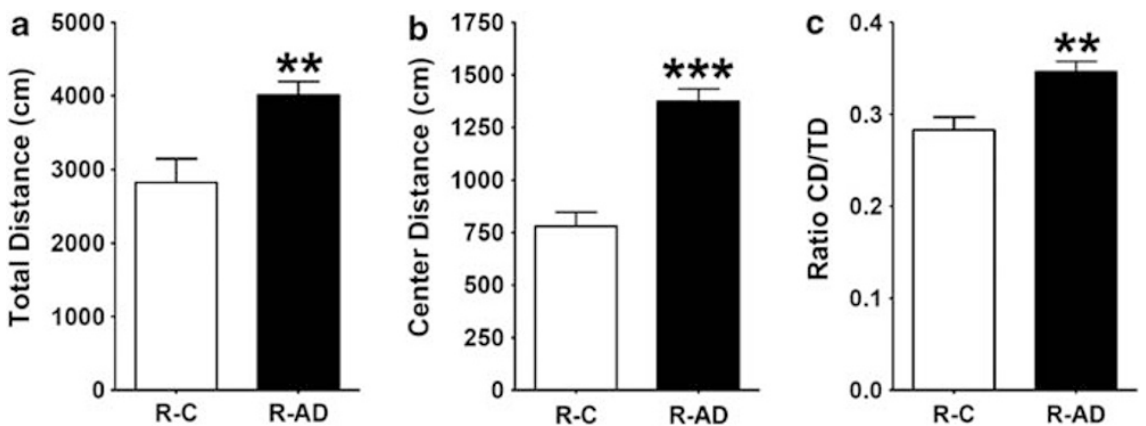

Figure 4 Interactions of recurrent restraint stress (RRS), short-term antidepressant treatment and fat diet on locomotor activity and anxiety. Open field tests were conducted after the period of RRS ended, each session lasted 60 min from days 14 to 67. (a) RRS-antidepressant treated group (R-AD) had increased total distance (TD) when compared with untreated RRS animals (R-C). (b) R-AD group had increased center distance (CD) when compared with R-C. (c) Anxiety was estimated by the ratio of center distance (CD) over TD; R-AD animals were significantly less anxious than R-C. Columns and bars (a-c) depict mean and s.e., respectively; ${ }^{* *} P<0.01 ;{ }^{* *} P<0.0001$.

significant body size and weight gain 122 days after discontinuation of antidepressant treatment in the context of a high-fat diet. We also show that a high-fat diet leads to correction of stress-induced weight loss in the absence of antidepressant treatment. These data suggest that exposure to antidepressants is a longterm risk factor for weight gain and obesity, even after antidepressant treatment is discontinued for a long time.

The relationship of antidepressant treatment and weight gain needs to be carefully examined. Clinically, weight gain is a common occurrence during both acute and long-term treatment with antidepressants, ${ }^{22}$ and it is a major problem for two reasons: (1) depressed patients are already at increased risk of cardiovascular disease, ${ }^{23}$ and weight gain could worsen that risk; and (2) weight gain is a cause of decreased antidepressant treatment compliance, resulting in treatment dropout. ${ }^{24}$ In adults, pooled analyses showed that SSRI treatment induces short-term weight loss, but long-term weight gain. ${ }^{25}$ There is still controversy of whether weight gain is mechanistically related to clinical improvement or if it is merely an undesirable side effect of antidepres- 
sant medication. ${ }^{22}$ Weight data have been derived primarily from re-analysis of drug trials or crosssectional observation data. ${ }^{26,27}$ Moreover, it appears that specific types of antidepressants, such as tricyclic antidepressants and mirtazapine, are more likely to induce weight gain. ${ }^{28}$

Our data suggest that antidepressant treatment has a complex effect on BW regulation dependent on environmental factors. Under standard laboratory conditions and diet, non-stressed rats receiving chronic fluoxetine treatment weighed less than saline-treated controls, which is compatible with the literature. ${ }^{15}$ In our stress-antidepressant-diet study, animals submitted to RRS consumed fewer calories and were lighter during the stress period.

What is reported here as a novel finding is that animals that were given fat diet after the stress period ended achieved full weight recovery: the weights of non-treated animals (R-C, saline control group) were not significantly different from those of non-stressed animals receiving fat diet (NR-CF). In contrast, non-treated rats receiving regular chow might not recover restraint-induced weight loss. ${ }^{29}$ RSS animals that received antidepressant treatment (R-AD) had behavioral manifestations of antidepressant action, as documented by decreased behavior that is attributable to stress and anxiety in open field. Our most noteworthy and novel finding was that when the combination of stress and short-term antidepressant treatment was followed by long-term high-fat diet after antidepressants were discontinued (Figures 1a and b), animals gained more weight when compared with rats submitted to the same paradigm but never exposed to antidepressants (R-C). As our open field data do not support a reduction of locomotor activity in the group exposed to antidepressants (Figure 4), the small but significant weight increase in those animals can be explained by their small but significantly higher caloric intake when compared with stressed animals not exposed to antidepressants.

Weight gain in the post-stress period is related to recovery of sympathetic function and hypothalamuspituitary-adrenal axis activation. ${ }^{30,31}$ Our data support the notion that the interaction of short-term antidepressant treatment during acute stress, and high-fat diet during long-term recovery, may potentiate the mechanisms underlying post-stress weight recovery and result in small but significant increases in FI and BW. Therefore, our results indicate that passage of time constitutes a key factor for the effects of antidepressants in weight regulation. This phenomenon is in agreement with the concept of TDS described in detail by Antelman et al. ${ }^{18,32-34}$ Their studies demonstrate that antidepressant- or electroconvulsive shock-induced effects grow with the passage of time. ${ }^{35}$ For instance, a single electroconvulsive shock reduced dopamine autoreceptor sensitivity by $85 \%$ in the central nervous system after 1 week, but not after $1 \mathrm{~h} .{ }^{35}$ Furthermore, six daily electroconvulsive shocks produced a similar magnitude of inhibition after 1 week of the initial intervention as that displayed by a single electroconvulsive shock. ${ }^{35}$ Similar results were also achieved by the administration of tricyclic antidepressants such as imipramine and amitriptyline. ${ }^{35}$ The results of that body of work suggest that the observed delay in the therapeutic effects of antidepressant occurred as a consequence of the biological changes triggered by the initial treatment rather than pharmacokinetics factors elicited by daily chronic drug administration. ${ }^{35}$ It is plausible that the concept of TDS may be applied to the pharmacological effects of antidepressants in weight regulation, which would result in long-term increases in caloric intake and BW gain. We hypothesize that the mechanism of action for TDS might require epigenetic modifications.

This study suggests that the 'paradoxical' effect of antidepressant treatment in BW in humans when compared with rodents may be explained by the simplified experimental paradigm generally used in rodents, which has not accounted for environmental factors, such as stress and diet, or a meaningfully long follow-up period. We show here that the inclusion of environmental factors after antidepressant treatment may induce long-term weight gain in rodents; therefore, the default explanation that differences in metabolic and neuroendocrine pathways regulating appetite and energy metabolism are required to explain the discrepancy of antidepressant effects in BW between human and rodents ${ }^{14}$ needs to be revisited, and as such differences may in reality be small or non-existent.

Given that noncompliance to antidepressant treatment seems to be high in the general population $(>50 \%),{ }^{13}$ our data emphasize the need to examine whether these drugs have effects on BW regulation that persist on a long-term basis after their discontinuation. Alarmingly, SSRI drugs have become so popular that they are already been called 'lifestyle' drugs. ${ }^{36,37}$ In the United States, obesity rates started to increase markedly in the late 1980s, which coincides temporally with the introduction of SSRIs. Yearly increases in the rates of antidepressant dispensing $^{7}$ seem to parallel increases in obesity rates $;^{38}$ however, detailed analyses of those trends at the population level are required to establish a clinical association.

In conclusion, we propose here that antidepressant treatment, occurring in the context of conditions of high stress and high-fat intake, might be a covert, insidious and long-lasting contributor to weight gain, even after antidepressant drugs are discontinued for a long time, which is compatible with the phenomenon of TDS. Further research is needed to understand the pathways and pathophysiological mechanisms involved in pharmacological-environment interactions underlying the protracted effects of antidepressant drugs on caloric intake and body size and weight. Future studies should also address the role of stress and high-fat diets on long-term BW outcomes during and following antidepressant treatment in clinical 
settings. It would be particularly important to understand whether long-term BW gain also occurs in animals fed with a variety of different diets, following exposure to stress and time-limited antidepressant treatment.

\section{Conflict of interest}

The authors declare no conflict of interest.

\section{Acknowledgments}

This research was supported in part by the National Institutes of Health Grant K24RR017365 to MLW, and by institutional funds from the University of Miami and The Australian National University. The funders had no role in the study design, data collection and analysis, decision to publish or preparation of the manuscript. We thank Dr Sari Izenwasser for her invaluable help with behavioral experiments and setup.

\section{References}

1 Kessler RC, Chiu WT, Demler O, Merikangas KR, Walters EE. Prevalence, severity, and comorbidity of 12-month DSM-IV disorders in the National Comorbidity Survey Replication. Arch Gen Psychiatry 2005; 62: 617-627.

2 Kessler RC, McGonagle KA, Zhao S, Nelson CB, Hughes M, Eshleman S et al. Lifetime and 12-month prevalence of DSM-III-R psychiatric disorders in the United States. Results from the National Comorbidity Survey. Arch Gen Psychiatry 1994; 51: 8-19.

3 Vos T, Haby MM, Barendregt JJ, Kruijshaar M, Corry J, Andrews G. The burden of major depression avoidable by longer-term treatment strategies. Arch Gen Psychiatry 2004; 61: 1097-1103

4 Lopez AD, Murray CC. The global burden of disease, 1990-2020. Nat Med 1998; 4: 1241-1243.

5 Bulloch AG, Patten SB. Non-adherence with psychotropic medications in the general population. Soc Psychiatry Psychiatr Epidemiol 2010; 45: 47-56.

6 Wang PS, Simon G, Kessler RC. The economic burden of depression and the cost-effectiveness of treatment. Int J Methods Psychiatr Res 2003; 12: 22-33.

7 Milane MS, Suchard MA, Wong ML, Licinio J. Modeling of the temporal patterns of fluoxetine prescriptions and suicide rates in the United States. PLoS Med 2006; 3: e190.

8 Olfson M, Marcus SC. National patterns in antidepressant medication treatment. Arch Gen Psychiatry 2009; 66: 848-856.

9 Mant A, Rendle VA, Hall WD, Mitchell PB, Montgomery WS, McManus PR et al. Making new choices about antidepressants in Australia: the long view 1975-2002. Med J Aust 2004; 181: S21-S24.

10 Moore M, Yuen HM, Dunn N, Mullee MA, Maskell J, Kendrick T. Explaining the rise in antidepressant prescribing: a descriptive study using the general practice research database. BMJ 2009; 339: b3999.

11 Olie JP, Elomari F, Spadone C, Lepine JP. Antidepressants consumption in the global population in France. Encephale 2002; 28: 411-417.

12 Papakostas GI. Limitations of contemporary antidepressants: tolerability. J Clin Psychiatry 2007; 68(Suppl 10): 11-17.

13 Serna MC, Cruz I, Real J, Gasco E, Galvan L. Duration and adherence of antidepressant treatment (2003 to 2007) based on prescription database. Eur Psychiatry 2010; 25: 206
14 McAllister EJ, Dhurandhar NV, Keith SW, Aronne LJ, Barger J, Baskin $\mathrm{M}$ et al. Ten putative contributors to the obesity epidemic. Crit Rev Food Sci Nutr 2009; 49: 868-913.

15 File SE, Tucker JC. Behavioral consequences of antidepressant treatment in rodents. Neurosci Biobehav Rev 1986; 10 123-134.

16 Santarelli L, Saxe M, Gross C, Surget A, Battaglia F, Dulawa S et al. Requirement of hippocampal neurogenesis for the behavioral effects of antidepressants. Science 2003; 301: 805-809.

17 Wong ML, Licinio J. From monoamines to genomic targets: a paradigm shift for drug discovery in depression. Nat Rev Drug Discov 2004; 3: 136-151.

18 Antelman SM, Chiodo LA. Repeated antidepressant treatments induce a long-lasting dopamine autoreceptor subsensitivity: is daily treatment necessary for clinical efficacy? Psychopharmacol Bull 1981; 17: 92-94.

19 Brady LS, Gold PW, Herkenham M, Lynn AB, Whitfield Jr HJ. The antidepressants fluoxetine, idazoxan and phenelzine alter corticotropin-releasing hormone and tyrosine hydroxylase mRNA levels in rat brain: therapeutic implications. Brain Res 1992; 572: $117-125$.

20 Malberg JE, Eisch AJ, Nestler EJ, Duman RS. Chronic antidepressant treatment increases neurogenesis in adult rat hippocampus. J Neurosci 2000; 20: 9104-9110.

21 Yirmiya R, Pollak Y, Barak O, Avitsur R, Ovadia H, Bette M et al. Effects of antidepressant drugs on the behavioral and physiological responses to lipopolysaccharide (LPS) in rodents. Neuropsychopharmacology 2001; 24: 531-544.

22 Fava M. Weight gain and antidepressants. J Clin Psychiatry 2000; 61(Suppl 11): 37-41.

23 Glassman AH. Depression and cardiovascular comorbidity. Dialogues Clin Neurosci 2007; 9: 9-17.

24 Papakostas GI. Tolerability of modern antidepressants. J Clin Psychiatry 2008; 69(Suppl E1): 8-13.

25 Sussman N, Ginsberg DL, Bikoff J. Effects of nefazodone on body weight: a pooled analysis of selective serotonin reuptake inhibitor- and imipramine-controlled trials. J Clin Psychiatry 2001; 62: 256-260.

26 Cascade E, Kalali AH, Kennedy SH. Real-world data on SSRI antidepressant side effects. Psychiatry (Edgmont) 2009; 6: $16-18$.

$27 \mathrm{Hu}$ XH, Bull SA, Hunkeler EM, Ming E, Lee JY, Fireman B et al. Incidence and duration of side effects and those rated as bothersome with selective serotonin reuptake inhibitor treatment for depression: patient report versus physician estimate. J Clin Psychiatry 2004; 65: 959-965.

28 Schwartz TL, Nihalani N, Jindal S, Virk S, Jones N. Psychiatric medication-induced obesity: a review. Obes Rev 2004; 5: 115-121.

29 Chotiwat C, Harris RB. Antagonism of specific corticotropinreleasing factor receptor subtypes selectively modifies weight loss in restrained rats. Am J Physiol Regul Integr Comp Physiol 2008; 295: R1762-R1773.

30 Garcia A, Marti O, Valles A, Dal-Zotto S, Armario A. Recovery of the hypothalamic-pituitary-adrenal response to stress. Effect of stress intensity, stress duration and previous stress exposure. Neuroendocrinology 2000; 72: 114-125.

31 Chotiwat C, Kelso EW, Harris RB. The effects of repeated restraint stress on energy balance and behavior of mice with selective deletion of CRF receptors. Stress 2010; 13: 203-213.

32 Antelman SM, DeGiovanni LA, Kocan D, Perel JM, Chiodo LA. Amitriptyline sensitization of a serotonin-mediated behavior depends on the passage of time and not repeated treatment. Life Sci 1983; 33: 1727-1730.

33 Lace JW, Antelman SM. Cortical beta-adrenergic subsensitivity after desmethylimipramine may depend on the passage of time rather than daily treatment. Brain Res 1983; 278 359-361.

34 Antelman SM, Levine J, Gershon S. Time-dependent sensitization: the odyssey of a scientific heresy from the laboratory to the door of the clinic. Mol Psychiatry 2000; 5: 350-356.

35 Chiodo LA, Antelman SM. Electroconvulsive shock: progressive dopamine autoreceptor subsensitivity independent of repeated treatment. Science 1980; 210: 799-801. 
36 Atkinson T. Lifestyle drug market booming. Nat Med 2002; 8: 909.

37 Licinio J, Wong ML. Depression and obesity treatments are life saving. Nat Med 2002; 8: 1336.

38 CDC. Behavioral Risk Factor Surveillance System Survey Data. US Department of Health and Human Services, Centers for Disease Control and Prevention: Atlanta, GA, 2008.

This work is licensed under the Creative

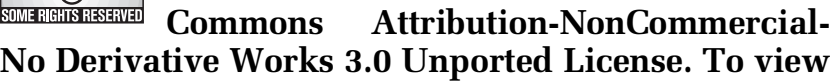
a copy of this license, visit http://creativecommons. org/licenses/by-nc-nd/3.0/ 